JOURNAL OF

SYMPLECTIC GEOMETRY

Volume 7, Number 2, 51-76, 2009

\title{
BERNSTEIN POLYNOMIALS, BERGMAN KERNELS AND TORIC KÄHLER VARIETIES
}

\author{
Steve ZeLDitch
}

\begin{abstract}
We show that the classical Bernstein polynomials $B_{N}(f)(x)$ on the interval $[0,1]$ (and their higher dimensional generalizations on the simplex $\Sigma_{m} \subset \mathbb{R}^{m}$ ) may be expressed in terms of Bergman kernels for the Fubini-Study metric on $\mathbb{C P}^{m}: B_{N}(f)(x)$ is obtained by applying the Toeplitz operator $f\left(N^{-1} D_{\theta}\right)$ to the Fubini-Study Bergman kernels. The expression generalizes immediately to any toric Kähler variety and Delzant polytope, and gives a novel definition of Bernstein "polynomials" $B_{h^{N}}(f)$ relative to any toric Kähler variety. They uniformly approximate any continuous function $f$ on the associated polytope $P$ with all the properties of classical Bernstein polynomials. Upon integration over the polytope, one obtains a complete asymptotic expansion for the Dedekind-Riemann sums $\frac{1}{N^{m}} \sum_{\alpha \in N P} f\left(\frac{\alpha}{N}\right)$ of $f \in C^{\infty}\left(\mathbb{R}^{m}\right)$, of a type similar to the Euler-MacLaurin formulae.
\end{abstract}

\section{Introduction}

Our starting point is the observation that the classical Bernstein polynomials

$$
B_{N}(f)(x)=\sum_{\alpha \in \mathbb{N}^{m}:|\alpha| \leq N}\left(\begin{array}{c}
N \\
\alpha
\end{array}\right) x^{\alpha}(1-\|x\|)^{N-|\alpha|} f\left(\frac{\alpha}{N}\right),
$$

on the $m$-simplex $\Sigma_{m} \subset \mathbb{R}^{m}$ may be expressed in terms of the BergmanSzegö kernels $\Pi_{h_{\mathrm{FS}}^{N}}(z, w)$ for the Fubini-Study metric on $\mathbb{C P}^{m}$ : Let $e^{i \theta}$ denote the standard $\mathbf{T}^{m}=\left(S^{1}\right)^{m}$ action on $\mathbb{C}^{m}$ and and let $D_{\theta_{j}}$ denote the linearization (or "quantization") of its infinitesimal generators on $H^{0}\left(\mathbb{C P}^{m}, \mathcal{O}(N)\right)$. As will be shown in Section 2 (see also Section 4),

$$
B_{N}(f)(x)=\left.\frac{1}{\Pi_{h_{\mathrm{FS}}^{N}}(z, z)} f\left(N^{-1} D_{\theta}\right) \Pi_{h_{\mathrm{FS}}^{N}}\left(e^{i \theta} z, z\right)\right|_{\theta=0, z=\mu_{h_{\mathrm{FS}}}^{-1}(x)},
$$

where $f \in C_{0}^{\infty}\left(\mathbb{R}^{m}\right)$. Here, $\Pi_{h_{\mathrm{FS}}^{N}}$ denotes the Bergman-Szegö kernel on powers $\mathcal{O}(N) \rightarrow \mathbb{C P}^{m}$ of the invariant hyperplane line bundle, $f\left(N^{-1} D_{\theta}\right)$ is 
defined by the spectral theorem and $\mu_{h_{\mathrm{FS}}}$ is the moment map corresponding to $h_{\mathrm{FS}}$. Thus, the Bernstein polynomial $B_{N} f(x)$ is the Berezin covariant symbol of the Toeplitz operator $\Pi_{h_{\mathrm{FS}}^{N}} f\left(N^{-1} D_{\theta}\right) \Pi_{h_{\mathrm{FS}}^{N}}$, i.e., the quotient $\frac{\mathcal{N}_{h_{\mathrm{FS}}^{N}}(z, z)}{\Pi_{h_{\mathrm{FS}}^{N}}(z, z)}$ of its kernel $\mathcal{N}_{h_{\mathrm{FS}}^{N}}(z, z)$ on the diagonal by the Bergman-Szegö on the diagonal. From this formula, many properties of Bernstein polynomials may be derived from properties of the Fubini-Study Bergman-Szegö kernel.

Furthermore, the formula (1.2) generalizes immediately to any polarized toric Kähler variety $(L, M, \omega)$ and defines analogs $B_{h^{N}}(f)(x)$ of Bernstein polynomials for any Delzant poytope $P$ and any positively curved toric hermitian metric $h$ on the invariant line bundle associated to $P$. We simply replace the Hermitian line bundle $\mathcal{O}(1) \rightarrow \mathbb{C P}^{m}$ with its Fubini-Study metric by any toric invariant Hermitian line bundle $(L, h) \rightarrow(M, \omega)$ (see Definition 3.1).

The connection between Bernstein polynomials and Bergman-Szegö kernels may be used to obtain asymptotic expansions of Bernstein polynomials as the degree $N \rightarrow \infty$;

Theorem 1.1. Let $(L, h) \rightarrow(M, \omega)$ be a toric Hermitian invariant line bundle over a toric Kähler variety with associated moment polytope P. Let $f \in C_{0}^{\infty}\left(\mathbb{R}^{m}\right)$ and let $B_{h^{N}}(f)(x)$ denote its Bernstein polynomial approximation in the sense of Definition 3.1. Then there exists a complete asymptotic expansion,

$$
\begin{aligned}
B_{h^{N}}(f)(x)= & f(x)+\mathcal{L}_{1} f(x) N^{-1}+\mathcal{L}_{2} f(x) N^{-2}+\cdots+\mathcal{L}_{m} f(x) N^{-m} \\
& +O\left(N^{-m-1}\right)
\end{aligned}
$$

in $C^{\infty}(\bar{P})$, where $\mathcal{L}_{j}$ is a differential operator of order $2 j$ depending only on curvature invariants of the metric $h$; the expansion may be differentiated any number of times.

In the case of classical Bernstein polynomials (1.1) (i.e., the interval or simplex), this expansion has recently been derived by Hörmander [18] by a different method (see (2.2)). The approach taken here is to use the Boutet de Monvel-Sjöstrand approximations of Bergman-Szegö kernels, with some simplifications in the case of toric hermitian metrics $[\mathbf{8}, \mathbf{2 4}]$. The operators $\mathcal{L}_{j}$ are computable from the coefficients of the asymptotic expansion of the Bergman-Szegö kernel $\Pi_{h^{N}}(z, z)$ on the diagonal in $[\mathbf{2 2}, \mathbf{3 2}]$. It should be noted that for general toric Hermitian line bundles, the Bernstein "polynomials" are not quite polynomials in the usual sense, although they are algebro-geometric objects in the sense of $[\mathbf{1 0}, \mathbf{3 1}]$; see Section 3 for further discussion. 
As defined in (1.2) and in Definition 3.1, the Bernstein polynomials are quotients

$$
B_{h^{N}}(f)(x)=\frac{\mathcal{N}_{h^{N}} f(x)}{\Pi_{h^{N}}\left(\mu_{h}^{-1}(x), \mu_{h}^{-1}(x)\right)}
$$

of a numerator polynomial $\mathcal{N}_{h^{N}} f(x)$ by the denominator $\Pi_{h^{N}}(z, z)$ with $\mu_{h}(z)=x$. Here, $\mu_{h}$ is the moment map associated to the Kähler form $\omega_{h}$ associated to $h$. The numerator polynomials also admit complete asymptotic expansions, and indeed the Bernstein polynomial expansions are derived from the numerator expansion and from the asymptotic expansion of the denominator. Hence, Theorem 1.1 follows from:

Theorem 1.2. With the same assumptions as above, there exist differential operators $\mathcal{N}_{j}$, such that

$$
\mathcal{N}_{h^{N}}(f)(x) \sim \frac{N^{m}}{\pi^{m}}\left(f(x)+N^{-1} \mathcal{N}_{1} f(x)+\cdots\right),
$$

where the operators $\mathcal{N}_{j}$ are computable from the Bergman kernel expansion for $\Pi_{h^{N}}(z, z)$. In particular,

$$
\mathcal{N}_{1} f(x)=\frac{1}{2}\left(f(x) S\left(\mu_{h}^{-1}(x)\right)+\nabla \mu_{h}\left(\mu_{h}^{-1}(x)\right) \cdot \nabla^{2} f(x)\right),
$$

where $S(z)$ is the scalar curvature of the Kähler metric $\omega_{h}$.

The operator $\mathcal{N}_{1}$ is calculated in Section 6 . Note that $S$ is constant on $\mu^{-1}(x)$, so it may be evaluated at any point of this set.

Theorem 1.2 has an application to Dedekind-Riemann sums over lattice points in dilates of the polytope $P$, i.e., sums of the form

$$
\sum_{\alpha \in N P} f\left(\frac{\alpha}{N}\right), \quad f \in C_{0}^{\infty}\left(\mathbb{R}^{m}\right) .
$$

Upon integration of $\mathcal{N}_{h^{N}} f(x)$ over $P$ and multiplication by a universal constant, one obtains:

Corollary 1.3. Let $f \in C_{0}^{\infty}\left(\mathbb{R}^{m}\right)$. Then there exist differential operators $\mathcal{E}_{j}$, such that

$$
\begin{aligned}
& \sum_{\alpha \in N P} f\left(\frac{\alpha}{N}\right) \sim N^{m} \int_{P} f(x) d x+\frac{N^{m-1}}{2} \int_{\partial P} f(x) d \sigma \\
& \quad+N^{m-2} \int_{P} \mathcal{E}_{2} f(x) d x+\cdots,
\end{aligned}
$$

where $\sigma$ is the Leray measure on $\partial P$ corresponding to the affine defining functions $\ell_{r}(x)=\left\langle x, \nu_{r}\right\rangle$ of the boundary facts (cf. 3.1). That is, on the rth facet of $\partial P, d \ell_{r} \wedge d \sigma=d x$. 
Exact and asymptotic formulae for $\sum_{\alpha \in \mathrm{NP}} f\left(\frac{\alpha}{N}\right)$ have been previously proved for special $f$ using the generalized Euler-MacLaurin formulae of Khovanskii-Pukhlikov, Brion-Vergne, Guillemin-Sternberg and others (cf. $[\mathbf{1 5}-\mathbf{1 7}, \mathbf{1 9}])$. For purposes of comparison, Theorem 4.2 of [16] states that for $f \in C_{0}^{\infty}\left(\mathbb{R}^{n}\right)$,

$$
\begin{aligned}
& \frac{1}{N^{m}} \sum_{\substack{\alpha \in \mathbb{N}^{m}:|\alpha| \leq N\\
}} f\left(\frac{\alpha}{N}\right) \sim\left(\sum_{F} \sum_{\gamma \in \Gamma_{F}^{1}} \tau_{\gamma}\left(\frac{1}{N} \frac{\partial}{\partial h}\right) \int_{P_{h}} f(x) d x\right) \\
& \quad \times\left.\right|_{h=0}+O\left(N^{-\infty}\right),
\end{aligned}
$$

where the sums involve various data associated to the polytope $P$ and where $P_{h}$ is a parallel dilate of $P$. We refer to $[\mathbf{1 6}]$ for the notation. The two-term expansion given in Corollary 1.3 was stated in [28]. It is straightforward to generalize the formula and proof to the case where $f$ is a symbol as in [17], and to obtain remainder estimates in the expansion.

A significant difference between the Euler-MacLaurin and the Bernstein methods for obtaining expansions of Dedekind-Riemann sums $\sum_{\alpha \in \mathbb{N}^{m}:|\alpha| \leq N}$ $f\left(\frac{\alpha}{N}\right)$ is that the Bernstein approaches uses an arbitrary toric Kähler metric while the Euler-MacLaurin approach is metric independent. This reflects the fact that the Bernstein approach is to integrate the pointwise expansion of Theorem 1.2, which depends on the metric $h$. The metric independence of the expansion in Corollary 1.3 is equivalent to a sequence of integration by parts identities involving curvature invariants. For instance, we obtain the second term in the expansion in Section 7 by using an integration by parts identity on polytopes due to Donaldson [12]; see also Section 2 for the simplest case. Conversely, comparison of the metric expansion in Theorem 1.2 and the Euler-MacLaurin expansion in (1.4) gives another proof of this identity, and generates further identities in the lower order terms for any choice of toric hermitian metric.

The connection between Bernstein polynomials, Bergman kernels and Berezin symbols appears to be new, and one of the principal motivations of this article is simply to point out the toric geometry underlying the classical Bernstein polynomials. We then exploit it to simplify the approximation theory and to extend it to general toric Kähler varieties. The generalized Bernstein approximation theory should be useful in the program of Yau-Tian-Donaldson of making algebro-geometric (i.e., polynomial) approximations to transcendental geometric objects on Kähler varieties (cf. $[\mathbf{1 1}, \mathbf{3 1}]$ ). For instance, in $[\mathbf{2 6}]$ what we recognize in this article as Bernstein polynomials were used to approximate geodesic rays in $C^{2}$ (see also $[\mathbf{2 3}]$ ). However, in [26], the function denoted by $f$ in Definition 3.1 in Section 3 (denoted $\mathcal{R}_{N}$ in [26]) also depended 
on $N$ in a subtle way, and so the polynomial approximations were much more complicated than the Bernstein polynomials of this article. One of our purposes in this article is to separate out the role of Bernstein polynomial approximations implicit in [26] for other applications, for instance to test configuration geodesics in [27]. In particular, the measures $\mu_{N}^{z}$ defined in (3.9) are studied in detail in [27], and the "law of large numbers" in Corollary 3.4 is developed into a large deviations principle.

We close the introduction with references to related work. As mentioned above, the article [18] also concerns relations between Bernstein polynomials and Bergman kernels, for the opposite purpose of deriving Bergman kernel expansions on Reinhardt domains from classical Bernstein polynomial expansions on the simplex. The exposition in Section 6 was influenced by its analysis of Bernstein polynomials. It also draws on the analysis of [24-26]. More on Berezin symbols of Toeplitz operators can be found in $[\mathbf{9 , 2 5}]$ and specifically in the toric case in [24].

Bernstein introduced Bernstein polynomials to uniformly approximate continuous functions on $[0,1][\mathbf{5}-\mathbf{7}]$. Kac and Szasz introduced analogous analytic functions on $[0, \infty]$ to approximate continuous functions uniformly on compact subsets of $[0, \infty]$. In [14], it is explained that the Szasz analytic functions are also of the form (1.2), but with the Bargmann-Fock Bergman kernel replacing the Fubini-Study one. Feng then generalizes the results of this article to infinite volume toric varieties (e.g., Calabi-Yau toric varieties), and explains how the Szasz analytic function is the scaling limit towards the boundary of a toric Bergman polynomial.

In addition to the Bergman-toric generalization of Bernstein polynomials, there also exists a probabilistic generalization of Bernstein polynomial which replaces $\left(\begin{array}{l}N \\ \alpha\end{array}\right)$ by the weighted number of lattice paths from 0 to $\alpha$ with steps in the polytope $P$. This definition also coincides with the canonical one in the case of the Fubini-Study metrics on $\mathbb{C P}^{m}$ but in general gives a different class of polynomials defined on the simplex of probability measures on $\{1, \ldots, m\}$. In the case of the simplex $\Sigma_{m}=P$, both spaces are the same, but in general they are not. The relevant analysis could be obtained from [30]; we will not discuss these generalizations here. In [29], Tate defines yet another kind of Bernstein polynomial on a convex polytope, based on certain "Bernstein measures". They are closely related to the analysis in [18]. In his recent survey [13, Section 2.3], Donaldson discusses a number of problems and results on norms of monomials and measures on lattice points in convex polytopes which are closely related to the material in $[24,26,29]$.

We would like to thank H. Hezari for a careful reading of the article and for pointing out some notational inconsistencies and misprints in an earlier version. 


\section{Fubini-Study and classical Bernstein polynomials}

Let us begin by explaining in more detail the Bernstein-Bergman connection for the Fubini-Study metric in one complex dimension. We recall that Bernstein polynomials of one variable give canonical uniform polynomial approximations to continuous functions $f \in C([0,1])$ :

$$
B_{N}(f)(x)=\sum_{j=0}^{N}\left(\begin{array}{c}
N \\
j
\end{array}\right) f\left(\frac{j}{N}\right) x^{j}(1-x)^{N-j} .
$$

They have the special feature that they simultaneously uniformly approximate all derivatives of $f$ if $f \in C^{k}$, i.e., $B_{N}(f)^{(k)}(x) \rightarrow f^{(k)}(x)$ (cf. [21]), and if $f \in C^{\infty}$, there exists a complete asymptotic expansion ([18]; see also [1] $)$

$$
B_{N}(f)(x) \sim \sum_{\mu=0}^{\infty} L_{\mu}\left(x, \frac{d}{d x}\right) f(x) N^{-\mu}
$$

for certain polynomial differential operators $L_{\mu}\left(x, \frac{d}{d x}\right)$,

$$
L_{0}=1, \quad L_{1}=\frac{1}{2}\left(x-x^{2}\right) \frac{d^{2}}{d x^{2}}, \quad L_{2}=\frac{1}{6}\left(x-x^{2}\right)(1-2 x) \frac{d^{3}}{d x^{3}}+\frac{1}{8}\left(x-x^{2}\right)^{2} \frac{d^{4}}{d x^{4}} .
$$

In this case, $B_{N}(f)=\frac{1}{N+1} \mathcal{N}_{N}(f)$ (cf. Theorem 1.2), and also

$$
\left(\begin{array}{c}
N \\
j
\end{array}\right) \int_{0}^{1} x^{j}(1-x)^{N-j} d x=\left(\begin{array}{c}
N \\
j
\end{array}\right) \frac{j !(N-j) !}{(N+1) !}=\frac{1}{N+1} .
$$

Hence, (2.1) implies that

$$
\begin{aligned}
\int_{0}^{1} & \mathcal{N}_{N}(f)(x) d x \\
& =\sum_{j=0}^{N} f\left(\frac{j}{N}\right) \\
& =(N+1)\left(\int_{0}^{1} f(x) d x+\frac{1}{2 N} \int_{0}^{1}\left(x-x^{2}\right) f^{\prime \prime}(x) d x+\cdots\right) \\
& =(N+1)\left(\int_{0}^{1} f(x) d x+\frac{1}{2 N}\left(f(1)-f(0)-2 \int_{0}^{1} f(x) d x\right)+\cdots\right) \\
& =N \int_{0}^{1} f(x) d x+\frac{1}{2}(f(1)-f(0))+O\left(\frac{1}{N}\right) .
\end{aligned}
$$

We included the routine details to point out that obtaining the first two terms of the Euler-MacLaurin Riemann sum expansion in Corollary 1.3 required two integrations by parts and cancellations of $\int_{0}^{1} f(x) d x$ in the constant term between the subleading term of the dimension (Riemann-Roch) 
polynomial $(N+1)$ term and in the $\int_{0}^{1} L_{1} f(x) d x$ term. Similar cancellations occur in the general case (see the proof of Theorem 1.3).

We now relate the Bernstein polynomials $B_{N}(f)$ on $[0,1]$ to the Bergman kernel for the Fubini-Study metric on $\mathbb{C P}^{1}$. The discussion is almost the same for the $m$-simplex $\Sigma_{m} \subset \mathbb{R}^{m}$ and the Bergman kernel for the Fubini metric on $\mathbb{C P}^{m}$, so we carry it out in all dimensions. We first need to recall some standard facts about the Bergman or Szegö kernels for the FubiniStudy metric.

By the $m$-simplex we mean the convex set $\Sigma_{m}=\left\{\left(x_{1}, \ldots, x_{m}\right) \in \mathbb{R}_{+}^{m}\right.$ : $\left.\|x\|:=\sum_{j=1}^{m} x_{j} \leq 1\right\}$. We denote its dilate by $N \in \mathbb{N}$ by $N \Sigma_{m}$. As discussed in [24] and elsewhere (see [24] for references), the space $\operatorname{Poly}\left(N \Sigma_{m}\right)$ of polynomials with exponents $\alpha \in N \Sigma_{m}$ can be identified with the space of degree- $N$ homogeneous holomorphic polynomials in $m+1$ variables by identifying the (non-homogeneous) polynomial

$$
f\left(z_{1}, \ldots, z_{m}\right)=\sum_{|\alpha| \leq N} c_{\alpha} z^{\alpha} \quad\left(z^{\alpha}=z_{1}^{\alpha_{1}} \cdots z_{m}^{\alpha_{m}}\right)
$$

with the homogeneous polynomial

$$
F\left(\zeta_{0}, \ldots, \zeta_{m}\right)=\sum_{|\alpha| \leq N} c_{\alpha} \zeta_{0}^{N-|\alpha|} \zeta_{1}^{\alpha_{1}} \cdots \zeta_{m}^{\alpha_{m}}
$$

The space $\mathcal{P} \operatorname{oly}\left(N \Sigma_{m}\right)$ has a natural $\mathcal{L}^{2}$ inner product,

$$
\langle f, \bar{g}\rangle=\frac{1}{m !} \int_{S^{2 m+1}} F \bar{G} d \nu,
$$

where $d \nu$ is normalized Haar measure on $S^{2 m+1}$.

This inner product is equivalent to viewing $f, g$ as a holomorphic sections of the $N$ th power $\mathcal{O}(N)$ of the hyperplane line bundle $\mathcal{O}(1) \rightarrow \mathbb{C P}^{m}$ dual to the tautological line bundle. The line bundle $\mathcal{O}(1)$ carries a natural metric $h_{\mathrm{FS}}$ given by

$$
\|s\|_{h_{\mathrm{FS}}}([w])=\frac{|(s, w)|}{|w|}, \quad w=\left(w_{0}, \ldots, w_{m}\right) \in \mathbb{C}^{m+1},
$$

for $s \in \mathbb{C}^{m+1 *} \equiv H^{0}\left(\mathbb{C P}^{m}, \mathcal{O}(1)\right)$, where $|w|^{2}=\sum_{j=0}^{m}\left|w_{j}\right|^{2}$ and $[w] \in \mathbb{C P}^{m}$ denotes the complex line through $w$. The Kähler form on $\mathbb{C P}^{m}$ is the FubiniStudy form

$$
\omega_{\mathrm{FS}}=\frac{\sqrt{-1}}{2} \Theta_{h_{\mathrm{FS}}}=\frac{\sqrt{-1}}{2} \partial \bar{\partial} \log |w|^{2} .
$$

The natural Fubini-Study inner product on sections is then

$$
\left\langle s_{1}, s_{2}\right\rangle=\int_{\mathbb{C P} m}\left(s_{1}, s_{2}\right)_{h_{\mathrm{FS}}} \omega_{\mathrm{FS}}^{m} / m !
$$


In an affine chart and local frame $e$, sections have the form $f e$, where $f$ is a polynomial and the inner product takes the explicit form

$$
\langle f, \bar{g}\rangle=\frac{1}{m !} \int_{\mathbb{C}^{m}} \frac{f(z) \overline{g(z)}}{\left(1+\|z\|^{2}\right)^{N}} d V_{\mathrm{FS}}(z), \quad f, g \in \mathcal{P} \operatorname{oly}\left(N \Sigma_{m}\right),
$$

where $d V_{\mathrm{FS}}=\frac{\prod_{j=1}^{d} d z_{j} \wedge d \bar{z}_{j}}{\left(1+|z|^{2}\right)^{(d+1)}}$ is the Fubini-Study volume form. Both versions of the inner product generalize to any holomorphic line bundle.

A basis for $\mathcal{P} o l y\left(N \Sigma_{m}\right)$ is given by the monomials $\chi_{\alpha}(z)=z_{1}^{\alpha_{1}} \cdots z_{m}^{\alpha_{m}}$, $|\alpha| \leq N$. The monomials $\left\{\chi_{\alpha}\right\}$ are orthogonal but not normalized. Their $\mathcal{L}^{2}$ norms given by the inner product (2.4) are:

$$
\left\|\chi_{\alpha}\right\|=\left[\frac{(N-|\alpha|) ! \alpha_{1} ! \cdots \alpha_{m} !}{(N+m) !}\right]^{1 / 2} .
$$

Thus, an orthonormal basis for $\mathcal{P}$ oly $\left(N \Sigma_{m}\right)$ is given by the monomials

$$
\begin{aligned}
\frac{1}{\left\|\chi_{\alpha}\right\|} \chi_{\alpha} & =\left[\frac{(N+m) !}{(N-|\alpha|) ! \alpha_{1} ! \cdots \alpha_{m} !}\right]^{1 / 2} \chi_{\alpha}=\sqrt{\frac{(N+m) !}{N !}\left(\begin{array}{c}
N \\
\alpha
\end{array}\right)} \chi_{\alpha}, \\
|\alpha| & \leq N
\end{aligned}
$$

where

$$
\left(\begin{array}{l}
N \\
\alpha
\end{array}\right)=\frac{N !}{(N-|\alpha|) ! \alpha_{1} ! \cdots \alpha_{m} !} .
$$

We let $\widehat{\chi}_{\alpha}^{N}: S^{2 m+1} \rightarrow \mathbb{C}$ denote the homogenization of $\chi_{\alpha}$ :

$$
\widehat{\chi}_{\alpha}^{N}(x)=x_{0}^{N-|\alpha|} x_{1}^{\alpha_{1}} \cdots x_{m}^{\alpha_{m}} .
$$

The Bergman or Szegö kernel $\Pi_{h_{\mathrm{FS}}^{N}}$ for the Fubini-Study metric is the orthogonal projection to the space $H^{0}\left(\mathbb{C P}^{m}, \mathcal{O}(N)\right)$ of holomorphic sections with respect to the inner produced induced by $h_{\mathrm{FS}}$, which lifts to the orthogonal projection $\hat{\Pi}_{h_{\mathrm{FS}}^{N}}$ onto $\mathcal{P}$ oly $(N \Sigma)$. The latter is given by

$$
\hat{\Pi}_{h_{\mathrm{FS}}^{N}}(x, y)=\sum_{|\alpha| \leq N} \frac{1}{\left\|\chi_{\alpha}\right\|^{2}} \widehat{\chi}_{\alpha}(x) \overline{\widehat{\chi}_{\alpha}(y)}=\frac{(N+m) !}{N !}\langle x, \bar{y}\rangle^{N},
$$

for $x, y \in S^{2 m+1}$. In particular, on the diagonal we have $\langle x, x\rangle=1$ and

$$
\hat{\Pi}_{h_{\mathrm{FS}}^{N}}(x, x)=\frac{(N+m) !}{N !} .
$$

In terms of the standard local affine frame on $\mathbb{C}^{m}$, we have $\widehat{\chi}_{\alpha}^{N}(z)=$ $\frac{z^{\alpha}}{\left(1+\|z\|^{2}\right)^{N / 2}}$, and hence

$$
\Pi_{h_{\mathrm{FS}}^{N}}(z, w)=\frac{(N+m) !}{N !} \frac{\sum_{|\alpha| \leq N}\left(\begin{array}{c}
N \\
\alpha
\end{array}\right) z^{\alpha} \bar{w}^{\alpha}}{\left(1+\|z\|^{2}\right)^{N / 2}\left(1+\|w\|^{2}\right)^{N / 2}} .
$$


We now have the ingredients to identify Bernstein polynomials for the simplex $N \Sigma_{m}$ in terms of the Fubini-Study Bergman-Szegö kernel. The Kähler potential of the Fubini-Study metric is $\varphi_{\mathrm{FS}}=\log \left(1+\|z\|^{2}\right)$, where $\|z\|^{2}=\sum_{j}\left|z_{j}\right|^{2}$, and its moment map is

$$
\mu_{h_{\mathrm{FS}}}(z)=\left(\frac{\left|z_{1}\right|^{2}}{1+\|z\|^{2}}, \ldots, \frac{\left|z_{m}\right|^{2}}{1+\|z\|^{2}}\right) .
$$

The Fubini-Study symplectic potential is the convex function on $\Sigma_{m}$ given by the Legendre transform of $\varphi_{\mathrm{FS}}$ in logarithm coordinates,

$$
u_{0}(x)=\sum_{j=1}^{m} x_{j} \log x_{j}+(1-\|x\|) \log (1-\|x\|),
$$

where $\|x\|=\sum_{j=1}^{m} x_{j}$. A simple calculation shows that the Bernstein terms may be expressed in terms of the symplectic potential as

$$
\left(\begin{array}{c}
N \\
\alpha
\end{array}\right) x^{\alpha}(1-\|x\|)^{N-|\alpha|}=\frac{N !}{(N+m) !} \frac{e^{N\left(u_{0}(x)+\left\langle(\alpha / N)-x, \nabla u_{0}(x)\right\rangle\right)}}{\left\|z^{\alpha}\right\|_{h_{\mathrm{FS}}^{N}}^{2}} .
$$

It follows that the Fubini-Study Bernstein polynomial is given by

$B_{N}(f)(x)=\frac{1}{\Pi_{h_{\mathrm{FS}}^{N}}(z, z)} \sum_{\alpha=0}^{N} f\left(\frac{\alpha}{N}\right) \frac{e^{N\left(u_{0}(x)+\left\langle(\alpha / N)-x, \nabla u_{0}(x)\right\rangle\right)}}{\left\|z^{\alpha}\right\|_{h_{\mathrm{FS}}^{N}}^{2}}, \quad z=\mu_{h_{\mathrm{FS}}}^{-1}(x)$.

On the other hand, one can also express the Bergman-Szegö kernel in terms of the symplectic potential at the points $\left(e^{i \theta} z, z\right)$ as

$$
\begin{aligned}
\Pi_{h_{\mathrm{FS}}^{N}}\left(e^{i \theta} z, z\right) & =\sum_{\alpha=0}^{N} e^{i\langle\theta, \alpha\rangle} \frac{e^{N\left(u_{0}(x)+\left\langle(\alpha / N)-x, \nabla u_{0}(x)\right\rangle\right)}}{\left\|z^{\alpha}\right\|_{h_{\mathrm{FS}}^{N}}^{2}} \\
& =\Pi_{h_{\mathrm{FS}}^{N}}(z, z) \sum_{\alpha=0}^{N}\left(\begin{array}{c}
N \\
\alpha
\end{array}\right) e^{i\langle\theta, \alpha\rangle} x^{\alpha}(1-\|x\|)^{N-|\alpha|} .
\end{aligned}
$$

Indeed, comparing (2.14) and (2.17), we see that the two expressions for the Bergman-Szegö kernel agree as long as

$$
\left|z^{\alpha}\right|^{2} e^{-N \log \left(1+\|z\|^{2}\right)}=e^{N\left(u_{0}(x)+\left\langle(\alpha / N)-x, \nabla u_{0}(x)\right\rangle\right)}, \quad \text { when } \mu_{h_{\mathrm{FS}}}(z)=x,
$$

and this follows from the pair of identities, $\left|z^{\alpha}\right|^{2}=e^{\left\langle\alpha, \nabla u_{0}(x)\right\rangle}, \log \left(1+|z|^{2}\right)=\left\langle x, \nabla u_{0}(x)\right\rangle-u_{0}(x) \quad$ when $\mu_{h_{\mathrm{FS}}}(z)=x$. On the open orbit, we may use logarithmic coordinates $z=e^{\rho / 2+i \theta}$. Then $\rho=\nabla u_{0}(x)$ and the identities are equivalent to the fact that the Kähler potential and symplectic potential are Legendre transforms of each other. Since both sides of (2.17) are continuous, the equality extends to all of $M$ and $\bar{P}$. 
Applying the operator $f\left(\frac{D_{\theta}}{N}\right)$ just replaces $e^{i\langle\theta, \alpha\rangle}$ by $f\left(\frac{\alpha}{N}\right)$. Then, dividing by $\Pi_{h_{\mathrm{FS}}^{N}}(z, z)$ gives $(2.16)$ and (1.2). Together with the formulae above for norms of monomials and the Szegö kernel in dimension $m$, the formula (1.1) also reduces to $(2.16)$.

\section{Definition of the generalized Bernstein polynomials}

We now generalize the definition of Bernstein polynomial to any polarized toric Kähler variety, and generalize the calculations of the previous section.

We recall that a toric Kähler manifold is a Kähler manifold $(M, J, \omega)$ on which the complex torus $\left(\mathbb{C}^{*}\right)^{m}$ acts holomorphically with an open orbit $M^{o}$. We assume that $M$ is projective and that $P$ is a Delzant polytope, i.e., a convex integral polytope in $\mathbb{R}^{m}$ with the property that each vertex is contained in exactly $m$ facets, and the normals to the $m$ facets at each vertex form a $\mathbb{Z}$-basis for a lattice $\Gamma \subset \mathbb{R}^{m}$ so that $\mathbf{T}^{m}=\mathbb{R}^{m} / \Gamma$ is the torus acting on $M_{P}$. The convex polytope $P$ is defined by a set of inequalities of

$$
\left\langle x, v_{r}\right\rangle \geq \lambda_{r}, \quad r=1, \ldots, d,
$$

where $v_{r}$ is a primitive element of the lattice and inward-pointing normal to the $r$ th $(n-1)$-dimensional face of $P$.

We denote by $\mathbf{T}^{m}=\left(S^{1}\right)^{m}$ the real torus underlying $\left(\mathbb{C}^{*}\right)^{m}$. By a toric Kähler metric we mean a Kähler metric $\omega$ invariant under $\mathbf{T}^{m}$. We assume that $\frac{1}{\pi} \omega$ is a de Rham representative of the Chern class $c_{1}(L) \in H^{2}(M, \mathbb{R})$ of the invariant holomorphic line bundle $L \rightarrow M$. We let $h$ denote the Hermitian metric on $L$ inducing the Chern connection with curvature $(1,1)$ form $\omega_{h}=\omega$. Here, given a Hermitian metric $h$,

$$
\omega_{h}=-\frac{\sqrt{-1}}{2} \partial \bar{\partial} \log \left\|e_{L}\right\|_{h}^{2},
$$

where $e_{L}$ denotes a local holomorphic frame (i.e., a nonvanishing section) of $L$ over an open set $U \subset M$, and $\left\|e_{L}\right\|_{h}=h\left(e_{L}, e_{L}\right)^{1 / 2}$ denotes the $h$-norm of $e_{L}$. We often write $\omega$ for $\omega_{h}$ when the metric is fixed.

Now fix a basepoint $m_{0}$ on the open orbit and identify $M^{o} \equiv\left(\mathbb{C}^{*}\right)^{m}$, endowing $M^{o}$ with the logarithmic coordinates

$$
z=e^{\rho / 2+i \varphi} \in\left(\mathbb{C}^{*}\right)^{m}, \quad \rho, \varphi \in \mathbb{R}^{m} .
$$

Over the open orbit, $\omega$ has a Kähler potential, i.e., $\omega=-2 i \partial \bar{\partial} \varphi(z)$. The associated Hermitian metric then has the form $h=e^{-\varphi}$. Invariance under the real torus action implies that $\varphi$ only depends on the $\rho$-variables, hence

$$
\omega=\frac{i}{2} \sum_{j, k} \frac{\partial^{2} \varphi}{\partial \rho_{k} \rho_{j}} \frac{d z_{j}}{z_{j}} \wedge \frac{d \bar{z}_{k}}{\bar{z}_{k}} .
$$

We sometimes subscript $\omega$ to indicate the associated hermitian metric or Kähler potential, e.g., $\omega=\omega_{h}=\omega_{\varphi}$. By a slight abuse of notation, we denote 
the Kähler potential in the logarithmic coordinates by $\varphi(\rho)$. Positivity of $\omega$ implies that $\varphi$ is strictly convex of $\rho \in \mathbb{R}^{n}$.

The real torus $\mathbf{T}^{m}$ acts on $(M, \omega)$ in a Hamiltonian fashion with respect to $\omega$, and its moment map $\mu_{\varphi}=\mu_{h}$ with respect to $\omega_{\varphi}=\omega_{h}$ is defined by

$$
\mu_{h}\left(z_{1}, \ldots, z_{m}\right)=\nabla_{\rho} \varphi\left(\rho_{1}, \ldots, \rho_{m}\right), \quad\left(z=e^{\rho / 2+i \theta}\right) .
$$

The symplectic potential $u_{\varphi}$ associated to the Kähler potential is defined to be the Legendre-dual of $\varphi$, defined as follows: for $x \in P$ there is a unique $\rho$ such that $\mu_{\varphi}\left(e^{\rho / 2}\right)=\nabla_{\rho} \varphi=x$. Then the Legendre transform is defined to be the convex function

$$
u_{\varphi}(x)=\langle x, \rho\rangle-\varphi(\rho), \quad e^{\rho / 2}=\mu_{\varphi}^{-1}(x)
$$

on $P$.

Guillemin [15] (see also [2]) has defined a "canonical" Kähler metric and symplectic potential, as follows: let $l_{r}: \mathbf{R}^{n} \rightarrow \mathbf{R}$ be the affine functions,

$$
\ell_{r}(x)=\left\langle x, v_{r}\right\rangle-\lambda_{r} .
$$

Then the canonical symplectic potential is defined by

$$
u_{0}(x)=\sum_{k} \ell_{k}(x) \log \ell_{k}(x),
$$

which in turn corresponds to a canonical Kähler potential. Every symplectic potential has the same singularities on the boundary $\partial P$ as the canonical symplectic potential.

In general, we denote by $G_{\varphi}=\nabla_{x}^{2} u_{\varphi}$ the Hessian of the symplectic potential. It has simple poles on $\partial P$. We also denote by $H_{\varphi}(\rho)=\nabla_{\rho}^{2} \varphi\left(e^{\rho / 2}\right)$ the Hessian of the Kähler potential on the open orbit in $\rho$ coordinates. By Legendre duality,

$$
H_{\varphi}(\rho)=G_{\varphi}^{-1}(x), \quad \mu_{\varphi}\left(e^{\rho / 2}\right)=x .
$$

We now let $(L, h) \rightarrow M$ denote the invariant Hermitian line bundle with curvature $\omega_{h}=\omega$. A natural basis of the space of holomorphic sections $H^{0}\left(M, L^{N}\right)$ associated to the $N$ th power of $L \rightarrow M$ corresponds to monomials $z^{\alpha}$ where $\alpha$ is a lattice point in the $N$ th dilate of the polytope, $\alpha \in N P \cap \mathbb{Z}^{m}$. The hermitian metric $h$ on $L$ induces inner products $\operatorname{Hilb}_{N}(h)$ on $H^{0}\left(M, L^{N}\right)$, defined by

$$
\left\langle s_{1}, s_{2}\right\rangle_{h^{N}}=\int_{M}\left(s_{1}(z), s_{2}(z)\right)_{h^{N}} \frac{\omega_{h}^{m}}{m !} .
$$

The monomials are orthogonal with respect to any such toric inner product and have the norm-squares

$$
Q_{h^{N}}(\alpha)=\int_{\mathbb{C}^{m}}\left|z^{\alpha}\right|^{2} e^{-N \varphi(z)} d V_{\varphi}(z)
$$


where $d V_{\varphi}=(i \partial \bar{\partial} \varphi)^{m} / m$ !. Integrating over the torus fibers, we obtain an expression in terms of the symplectic potential,

$$
Q_{h^{N}}(\alpha)=C_{L} \int_{P} e^{N\left(u_{\varphi}(x)+\left\langle(\alpha / N)-x, \nabla u_{\varphi}(x)\right\rangle\right.} d x,
$$

where $C_{L}$ is the (common) volume of the torus fibers. It depends on the expression for $\frac{\omega_{h}^{m}}{m !}$ in action-angle variables with respect to the moment map $\mu_{h}$, hence on the first Chern class $c_{1}(L)$.

The Bergman-Szegö kernels for this hermitian metric are the orthogonal projections with respect to $\operatorname{Hilb}_{N}(h)$ to $H^{0}\left(M, L^{N}\right)$. If we denote the sections corresponding to the monomials by $S_{\alpha}$, then

$$
\Pi_{h^{N}}(z, w)=\sum_{\alpha \in N P} \frac{S_{\alpha}(z) \otimes S_{\alpha}(w)^{*}}{Q_{h^{N}}(\alpha)} .
$$

The diagonal contraction of the kernel is denoted by $\Pi_{h^{N}}(z, z)=$ $\frac{\sum_{\alpha \in N P}\left\|S_{\alpha}(z)\right\|_{h^{N}}^{2}}{Q_{h^{N}}(\alpha)}$.

The following definition generalizes the formula of (1.1) to any toric Kähler manifold.

Definition 3.1. Let $f \in C(\bar{P})$. The $N$ th normalized (Bergman-)Bernstein polynomial approximation to $f$ with respect to the hermitian metric $h$ on $L \rightarrow M$ is defined by

$$
\left\{\begin{array}{l}
B_{h^{N}} f(x)=\frac{1}{\Pi_{h^{N}}(z, z)} \mathcal{N}_{h^{N}} f(x), \text { where } \\
\mathcal{N}_{h^{N}} f(x)=\sum_{\alpha \in N P} f\left(\frac{\alpha}{N}\right) \frac{e^{N\left(u_{\varphi}(x)+\left\langle(\alpha / N)-x, \nabla u_{\varphi}(x)\right\rangle\right)}}{Q_{h^{N}}(\alpha)} .
\end{array}\right.
$$

To our knowledge, the only previously studied cases are the Bernstein polynomials for the simplex (1.1) or the $d$-cube, where

$$
B_{N}(f)(x)=\sum_{0 \leq i_{1}, \ldots, i_{d} \leq N} f\left(\frac{i_{1}}{N}, \ldots, \frac{i_{d}}{N}\right) \prod_{k=1}^{d}\left(\begin{array}{l}
N \\
i_{k}
\end{array}\right) x_{k}^{i_{k}}\left(1-x_{k}\right)^{N-i_{k}} .
$$

Here, $\left(x_{1}, \ldots, x_{d}\right) \in[0,1]^{d}$.

3.1. Associated measures $\mu_{N}^{z}$. As in the classical case, Bernstein polynomials are closely related to certain probability measures on $\bar{P}$. We define

$$
\mu_{N}^{z}:=\sum_{\alpha \in N P} \frac{\mathcal{P}_{h^{N}}(\alpha, z)}{\Pi_{h^{N}}(z, z)} \delta_{\alpha / N},
$$

where $\mathcal{P}_{h^{N}}(\alpha, z)$ denote the Fourier coefficients of the Bergman kernel with respect to the $\mathbf{T}^{m}$,

$$
\mathcal{P}_{h^{N}}(\alpha, z):=\frac{\left|z^{\alpha}\right|^{2} e^{-N \varphi(z)}}{Q_{h^{N}}(\alpha)} .
$$


Proposition 3.2. Let $f \in C(\bar{P})$ and let $x=\mu_{\varphi}(z)$ and let $h=e^{-\varphi}$. Then,

$$
B_{h^{N}} f(x)=\int_{P} f(y) d \mu_{N}^{z}(y) .
$$

Proof. This follows from the pair of identities,

$$
\begin{aligned}
\int_{P} f(y) d \mu_{N}^{z}(y) & =\sum_{\alpha \in N P} f\left(\frac{\alpha}{N}\right) \frac{\mathcal{P}_{h^{N}}(\alpha, z)}{\Pi_{h^{N}}(z, z)} \\
& =\frac{1}{\Pi_{h^{N}}(z, z)} \sum_{\alpha \in N P} f\left(\frac{\alpha}{N}\right) \frac{e^{N\left(u_{\varphi}(x)+\left\langle(\alpha / N)-x, \log \mu_{\varphi}^{-1}(x)\right\rangle\right.}}{Q_{h^{N}}(\alpha)} .
\end{aligned}
$$

The first equality is obvious from the definition. The second equality generalizes the identity (2.18):

$$
\left|z^{\alpha}\right|^{2} e^{-N \varphi(z)}=e^{N\left(u_{\varphi}(x)+\left\langle(\alpha / N)-x, \log \mu_{\varphi}^{-1}(x)\right\rangle\right)}, \quad \text { when } \mu_{\varphi}(z)=x .
$$

As in the case of the Fubini-Study metric, the identity splits into two identities on the open orbit,

$$
\left|z^{\alpha}\right|^{2}=e^{\langle\alpha, \rho\rangle}, \quad e^{-N \varphi(z)}=e^{N\left(u_{\varphi}(x)-\left\langle x, \log \mu_{\varphi}^{-1}(x)\right\rangle\right)} .
$$

The first follows from the fact that

$$
\nabla_{x} u_{\varphi}(x)=\log \mu_{\varphi}^{-1}(x)=\rho,
$$

since by (3.4), $\nabla_{x} u_{\varphi}(x)=\rho+\left\langle x, \nabla_{x} \rho\right\rangle-\left\langle\nabla \varphi(\rho), \nabla_{x} \rho\right\rangle=\rho$, as $\nabla \varphi(\rho)=x$. The second then follows from the fact that $\varphi(\rho)$ and $u_{\varphi}(x)$ are Legendre duals. The identity of the proposition then extends by continuity to the closure.

As a simple corollary, we obtain one of the standard properties of Bernstein polynomials.

Corollary 3.3. Let $f \in C(\bar{P})$. Then $\min _{\bar{P}} f \leq B_{h^{N}}(f)(x) \leq \max _{\bar{P}} f$.

Furthermore, as an obvious consequence of the leading order asymptotics in Theorem 1.1, we have:

Corollary 3.4. In the weak topology of measures on $C(\bar{P}), \mu_{k}^{z} \rightarrow \delta_{\mu_{h}(z)}$.

In [27], it will be shown that in addition the sequence $\left\{\mu_{k}^{z}\right\}_{k=1}^{\infty}$ of probability measures satisfies a large deviations principle for each $z$ (which is uniform in $z$ ). 
3.2. Bernstein polynomials for the Guillemin Kähler metric. Let us calculate explicitly the numerator polynomials for the canonical symplectic potential (3.5) or Kähler form. We have,

$$
\nabla u_{0}(x)=\sum_{k}\left(\log \ell_{k}\right) v_{k}+\bar{v}, \quad \bar{v}=\sum_{k} v_{k} .
$$

Hence,

$$
\left\langle\frac{\alpha}{N}-x, \nabla u_{0}(x)\right\rangle=\sum_{k}\left\langle\frac{\alpha}{N}-x, v_{k}\right\rangle \log \ell_{k}+\left\langle\frac{\alpha}{N}-x, \bar{v}\right\rangle,
$$

and

$$
\begin{aligned}
e^{N\left(u_{0}(x)+\left\langle(\alpha / N)-x, \nabla u_{0}(x)\right\rangle\right)} & =e^{\langle\alpha-N x, \bar{v}\rangle} \prod_{k}\left(\ell_{k}(x)\right)^{N \ell_{k}(x)+\left\langle\alpha-N x, v_{k}\right\rangle} \\
& =e^{\langle\alpha-N x, \bar{v}\rangle} \prod_{k}\left(\ell_{k}(x)\right)^{-N \lambda_{k}+\left\langle\alpha, v_{k}\right\rangle},
\end{aligned}
$$

where in the last line we use that $\ell_{k}(x)-\left\langle x, v_{k}\right\rangle=-\lambda_{k}$. Hence, the numerator of the canonical Bernstein polynomial may be rewritten as

$$
\mathcal{N}_{h^{N}} f(x)=\sum_{\alpha \in N P} f\left(\frac{\alpha}{N}\right) \frac{1}{Q_{h_{\text {can }}^{N}}(\alpha)} e^{\langle\alpha-N x, \bar{v}\rangle} \prod_{k}\left(\ell_{k}(x)\right)^{-N \lambda_{k}+\left\langle\alpha, v_{k}\right\rangle},
$$

which closely resembles the classical cases (where also $\bar{v}=0)$. Here, $Q_{h_{\text {can }}^{N}}(\alpha)$ is the norming constant with respect to the canonical Hermitian metric, given by (3.8) where the symplectic potential is chosen to be the canonical one.

In general, the symplectic potential has the form

$$
u_{\varphi}(x)=u_{0}(x)+g_{\varphi}(x)=\sum_{k} \ell_{k}(x) \log \ell_{k}(x)+g_{\varphi}(x),
$$

where $g_{\varphi} \in C^{\infty}(\bar{P})$ is smooth up the boundary $[\mathbf{2 , 1 2 , 1 5}$. Hence the $\alpha$ term gets multiplied by the additional factor

$$
e^{N\left(g_{\varphi}(x)+\left\langle(\alpha / k)-x, \nabla g_{\varphi}(x)\right\rangle\right)} .
$$

\section{Bernstein polynomials, Toeplitz operators and Berezin symbols}

In this section, we prove formula (1.2) in the setting of general toric Kähler varieties. We use the notation and terminology of $[\mathbf{2 4}]$.

We state the general result in the following

Proposition 4.1. We have,

$$
\mathcal{N}_{h^{N}}(f)(x)=\left.\left(\hat{\Pi}_{h^{N}} f\left(N^{-1} D_{\theta}\right) \hat{\Pi}_{h^{N}}\right)\left(e^{i \theta} z, z\right)\right|_{\theta=0 ; \mu_{h}(z)=x} .
$$


Remark 4.2. We note that when $f \equiv 1$, then $\mathcal{N}_{h^{N}}(f)(x) \equiv \Pi_{h^{N}}(z, z)$ $\left(\right.$ with $\left.\mu_{h}(z)=x\right)$.

Proof. The proof is simply a matter of unwinding the definitions and using some basic Fourier analysis. The Bergman kernel is a section of the bundle $\left(L^{N}\right) \otimes\left(L^{N}\right)^{*} \rightarrow M \times M$. It is simpler to deal with scalar kernels, and so we lift the Bergman kernel to a kernel $\hat{\Pi}_{h^{N}}(x, y)$ on the unit circle bundle $\pi: X \rightarrow M$ with respect to $h$ in the dual line bundle $L^{*}$. In other words, $X=\partial D_{h}^{*}$ is the boundary of the unit disc bundle with respect to $h$ in the dual line bundle $L^{*}$. We use local product coordinates $x=(z, \theta) \in M \times S^{1}$ on $X$ where $x=e^{i \theta} \frac{e(z)}{\|e(z)\|}$ in terms of a local holomorphic frame $e(z)$ for $L$. When working on $M$ we tacitly use the representative of $\Pi_{h^{N}}$ relative to the frame $e(z)^{N}$ of $L^{N}$. For the sake of brevity, we will not review the definitions but refer to [24] for the relevant background.

The space $H^{0}\left(M, L^{N}\right)$ is naturally isomorphic to the space $\mathcal{H}_{N}^{2}(X)$ of CR holomorphic functions transforming by $e^{i N \theta}$ under the $S^{1}$ action of the circle bundle $X \rightarrow M$. The generator of the $S^{1}$ action is denoted by $\frac{\partial}{\partial \theta}$. We further denote by $s \rightarrow \hat{s}$ the lift of a section to an equivariant CR function and by $\hat{\Pi}_{h^{N}}(x, y)$ the lifted Szegö kernel, i.e., the orthogonal projection from $L^{2}(X) \rightarrow \mathcal{H}_{N}^{2}(X)$. The monomial sections $s_{\alpha}$ which equal $z^{\alpha}$ on the open orbit lift to equivariant functions $\hat{s}_{\alpha}$ on $X$.

By the standard linearization of geometric quantization (reviewed in this context in [24]), the $\mathbf{T}^{m}$ action lifts to $X$ as contact transformations of the Chern connection form associated to $h$. For the sake of completeness, let us recall the lift of the torus action to $\mathcal{H}_{N}^{2}(X)$, and its linearization on $H^{0}\left(M, L^{N}\right)$ : The generators $\frac{\partial}{\partial \theta_{j}}$ of the $\mathbf{T}^{m}$ action on $M$ lift to contact vector fields $\Xi_{1}, \ldots, \Xi_{m}$ on $X$ with respect to the vertical contact 1-form $\alpha$ satisfying $d \alpha=\pi^{*} \omega$. The horizontal lifts of the Hamilton vector fields $\xi_{j}$ are then defined by

$$
\pi_{*} \xi_{j}^{h}=\xi_{j}, \quad \alpha\left(\xi_{j}^{h}\right)=0,
$$

and the contact vector fields $\Xi_{j}$ are given by:

$$
\Xi_{j}=\xi_{j}^{h}+2 \pi i\left\langle\mu \circ \pi, \xi_{j}^{*}\right\rangle \frac{\partial}{\partial \theta}=\xi_{j}^{h}+2 \pi i(\mu \circ \pi)_{j} \frac{\partial}{\partial \theta},
$$

where $\mu$ is the moment map corresponding to $h$, and where $\xi_{j}^{*} \in \mathbb{R}^{m}$ is the element of the Lie algebra of $\mathbf{T}^{m}$ which acts as $\xi_{j}$ on $M$.

It follows that the vector fields act as differential operators on the CR Hardy spaces, $\Xi_{j}: \mathcal{H}_{N}^{2}(X) \rightarrow \mathcal{H}_{N}^{2}(X)$ satisfying

$$
\left(\Xi_{j} \hat{S}\right)(\zeta)=\left.\frac{\partial}{\partial \varphi_{j}} \hat{S}\left(e^{i \varphi} \cdot \zeta\right)\right|_{\varphi=0}, \quad \hat{S} \in \mathcal{C}^{\infty}(X)
$$


Furthermore, the generator of the $S^{1}$ action acts on these spaces and

$$
\frac{\partial}{\partial \theta}: \mathcal{H}_{N}^{2}(X) \rightarrow \mathcal{H}_{N}^{2}(X), \quad \frac{1}{i} \frac{\partial}{\partial \theta} \hat{s}_{N}=N \hat{s}_{N} \quad \text { for } \quad \hat{s}_{N} \in \mathcal{H}_{N}^{2}(X) .
$$

Since by (4.1), the operators $\Xi_{j}$ act by translating functions by the $\mathbf{T}^{m}$ action lifted to $X$, we henceforth denote $\frac{1}{i} \Xi_{j}$ by $D_{\theta_{j}}$. Then for $1 \leq j \leq m$, the lifted monomials $\hat{\chi}_{\alpha} \in \mathcal{H}_{N}^{2}(X)$ are joint eigenfunctions of these commuting operators,

$$
D_{\theta_{j}} \hat{\chi}_{\alpha}=\alpha_{j} \hat{\chi}_{\alpha}, \quad \forall \alpha \in N P .
$$

The dilation $P \rightarrow N P$ is best viewed in terms of constructing a conic set of eigenvalues in one higher dimension by adding the operator

$$
\hat{I}_{m+1}=\frac{1}{i} \frac{\partial}{\partial \theta}-\sum_{j=1}^{m} D_{\theta_{j}} .
$$

The monomials $\widehat{\chi}_{\widehat{\alpha}}$ are then the joint eigenfunctions of these $(m+1)$ commuting operators and we define the "homogenization" $\widehat{N P} \subset \mathbb{Z}^{m+1}$ of the lattice points in the polytope $N P$ to be the set of all lattice point $\widehat{\alpha}^{N}$ of the form

$$
\widehat{\alpha}^{N}=\widehat{\alpha}:=\left(\alpha_{1}, \ldots, \alpha_{m}, N-|\alpha|\right), \quad \alpha=\left(\alpha_{1}, \ldots, \alpha_{m}\right) \in N P \cap \mathbb{Z}^{m} .
$$

Given $f \in C^{\infty}\left(\mathbb{R}^{m}\right)$, we now define $f\left(D_{\theta}\right)$ on $L^{2}(X)$ by the spectral theorem for $m$ commuting operators, i.e.,

$$
f\left(D_{\theta}\right)=\int_{\mathbb{R}^{m}} \hat{f}(\xi) e^{i\left\langle\xi, D_{\theta}\right\rangle} d \xi, \quad \text { where }\left\langle\xi, D_{\theta}\right\rangle=\sum_{j=1}^{m} \xi_{j} D_{\theta_{j}} .
$$

We then have

$$
f\left(N^{-1} D_{\theta}\right) \hat{s}_{\alpha}=f\left(\frac{\alpha}{N}\right) \hat{s}_{\alpha}
$$

Since $\hat{\Pi}_{h^{N}}(\hat{z}, \hat{w})=\sum_{\alpha \in N P} \hat{s}_{\alpha}(\hat{z}) \overline{\hat{s}_{\alpha}(\hat{w})}$, where $\hat{z}$ (etc.) denotes any point of $X$ projecting to $z$ under $\pi$, we have

$$
f\left(N^{-1} D_{\theta}\right) \hat{\Pi}_{h^{N}}\left(e^{i \theta} \hat{z}, \hat{w}\right)=\sum_{\alpha \in N P} f\left(\frac{\alpha}{N}\right) \hat{s}_{\alpha}(\hat{z}) \overline{\hat{s}_{\alpha}(\hat{w})} .
$$

It follows that

$$
\left.f\left(N^{-1} D_{\theta}\right) \hat{\Pi}_{h^{N}}\left(e^{i \theta} \hat{z}, \hat{w}\right)\right|_{\hat{z}=\hat{w}}=\sum_{\alpha \in N P} f\left(\frac{\alpha}{N}\right)\left|\hat{s}_{\alpha}(\hat{z})\right|^{2} .
$$

The right hand side is constant along the orbits of the $S^{1}$ action and may be identified with a function of $z \in M$. On $M$, we have $\left|\hat{s}_{\alpha}(\hat{z})\right|^{2}=\left\|s_{\alpha}(z)\right\|_{h^{N}}^{2}$ and by Proposition 3.2 we obtain the definition of the numerator polynomials when we substitute $z=\mu_{h}^{-1}(x)$. This is equivalent to the statement in the proposition. 
To obtain the Bernstein polynomial formula, we divide by $\Pi_{h^{N}}(z, z)$. As mentioned above, the ratio is the Berezin covariant symbol of $\hat{\Pi}_{h^{N}} f\left(N^{-1} D_{\theta}\right) \hat{\Pi}_{h^{N}}$.

\section{Proof of Theorems 1.1 and 1.2}

We now use the Boutet de Monvel - Sjöstrand parametrix $[\mathbf{3}, \mathbf{4}, \mathbf{8}]$ to obtain a complete asymptotic expansion for the Bernstein polynomials from Proposition 4.1. There now exist many expositions of the construction and properties of this parametrix, so we will only briefly recall the essential elements in the case of toric varieties $[\mathbf{2 4 , 2 6}$. We also use the notation $x, y$ for points of $X$, hoping that no confusion with coordinates on $P$ will occur.

We first recall that, on the diagonal, the Bergman-Szegö kernel has a complete asymptotic expansion,

$$
\Pi_{h^{N}}(z, z)=\sum_{i=0}^{d_{N}}\left\|S_{i}^{N}(z)\right\|_{h_{N}}^{2}=\frac{N^{m}}{\pi^{m}}\left[1+a_{1}(z) N^{-1}+a_{2}(z) N^{-2}+\cdots\right],
$$

for certain smooth coefficients $a_{j}(z)$, of which the first two lower coefficients are

$$
\left\{\begin{array}{l}
a_{1}=\frac{1}{2} S \\
a_{2}=\frac{1}{3} \Delta S+\frac{1}{24}\left(|R|^{2}-4|\operatorname{Ric}|^{2}+3 S^{2}\right)
\end{array}\right.
$$

where $R$, Ric and $S$ denote the curvature tensor, the Ricci curvature and the scalar curvature of $\omega_{h}$, respectively, and $\Delta$ denotes the Laplace operator of $\left(M, \omega_{h}\right)$; see $[\mathbf{4}, \mathbf{8}, \mathbf{2 2}, \mathbf{3 2}]$.

Off the diagonal, we have the following expansion:

Proposition 5.1. For any $C^{\infty}$ positive hermitian line bundle $(L, h)$, there exists a semi-classical amplitude in the parameter $N^{-1}, s_{N}(z, w) \sim$ $N^{m} s_{0}(z, w)+N^{m-1} s_{1}(z, w)+\cdots$, such that

$$
\Pi_{h^{N}}(z, w)=e^{N(\varphi(z, w)-(1 / 2)(\varphi(z)+\varphi(w)))} s_{N}(z, w)+O\left(N^{-\infty}\right),
$$

where $\varphi$ is a smooth local Kähler potential for $h$, and where $\varphi(z, w)$ is the almost-analytic extension of $\varphi(z)=\varphi(z, \bar{z})$.

Since the local Kähler potentials (e.g., the Kähler potential on the open orbit) are invariant under the $\mathbf{T}^{m}$ action, they can be expressed in the form $F\left(|z|^{2}\right)$ where $F \in C^{\infty}(\mathbb{R})$. We denote by $F(z \cdot \bar{w})$ the almost analytic extension of $F$. Thus, we have: 
Proposition 5.2. For any hermitian toric positive line bundle over a toric variety, the Szegö kernel for the metrics $h_{\varphi}^{N}$ have the asymptotic expansions in a local frame on $M$,

$$
\Pi_{h^{N}}(z, w) \sim e^{N\left(F(z \cdot \bar{w})-(1 / 2)\left(F\left(\|z\|^{2}\right)+F\left(\|w\|^{2}\right)\right)\right)} A_{N}(z, w) \quad \bmod N^{-\infty},
$$

where $A_{N}(z, w) \sim\left(\frac{N}{\pi}\right)^{m}\left(1+\frac{a_{1}(z, w)}{N}+\cdots\right)$ is a semi-classical symbol of order $m$.

We now prove Theorems 1.1 and 1.2.

Proof. We apply the geometric quantizations of the torus action to get, by Definition 3.1,

$$
\left.e^{i\left\langle\xi, N^{-1} D_{\theta}\right\rangle} \Pi_{h^{N}}\left(e^{i \theta} z, w\right)\right|_{z=w ; \theta=0}=\sum_{\alpha \in N P \cap \mathbb{Z}^{m}} \frac{e^{i\left\langle N^{-1} \alpha, \xi\right\rangle}\left|z^{\alpha}\right|^{2} e^{-N F\left(|z|^{2}\right)}}{\mathcal{Q}_{h^{N}}(\alpha)} .
$$

By Proposition 4.1, we obtain $\mathcal{N}_{h^{N}} f(x)$ by integrating the right side against $\hat{f}(\xi)$. We note that in general $\left.e^{i\left\langle\xi, N^{-1} D_{\theta}\right\rangle} \psi\left(e^{i \theta} w\right)\right|_{\theta=0}=\left.\psi\left(e^{i(\theta+(\xi / N))} w\right)\right|_{\theta=0}=$ $\psi\left(e^{i \xi / N} w\right)$. Performing the same transformation on the parametrix gives

$$
\mathcal{N}_{h^{N}}(f)(x) \sim \int_{\mathbb{R}^{m}} \hat{f}(\xi) e^{N\left(F\left(e^{i N^{-1}} \xi|z|^{2}\right)-F\left(|z|^{2}\right)\right)} A_{N}\left(z, e^{i(\xi / N)} z\right) d \xi,
$$

where $\sim$ means that the difference is a function which decays rapidly in $N$ along with its derivatives. Such a remainder may be neglected if we only consider expansions modulo rapidly decaying functions of $N$.

We have,

$$
\begin{aligned}
F_{\mathbb{C}} & \left(e^{i N^{-1} \xi}|z|^{2}\right)-F\left(|z|^{2}\right) \\
& =\int_{0}^{1} \frac{d}{d t} F_{\mathbb{C}}\left(e^{i t N^{-1} \xi}|z|^{2}\right) d t \\
& =i N^{-1} \int_{0}^{1}\left\langle\nabla_{\xi} F\left(e^{i t N^{-1} \xi+\rho}\right), \theta\right\rangle d t \\
& =i N^{-1}\left\langle\nabla_{\xi} F\left(e^{\rho}\right),(i \xi)\right\rangle+(i N)^{-2} \int_{0}^{1}(t-1) \nabla_{\rho}^{2}\left(F\left(e^{i t N^{-1} \xi+\rho}\right)\right)(i \xi)^{2} / 2 d t \\
& =i N^{-1}\langle\mu(z), \xi\rangle+(i N)^{-2} \nabla_{\rho}^{2}\left(F\left(e^{\rho}\right)\right)(i \xi)^{2}+R_{3}(\xi, N, \alpha) \\
& =i N^{-1}\langle\mu(z), \xi\rangle+(i N)^{-2}\left\langle H_{z} \xi, \xi\right\rangle+N^{-2} R_{3}(\xi, N, z),
\end{aligned}
$$

where

$$
R_{3}(\xi, N, z):=N^{-3} \int_{0}^{1}(t-1)^{2} \nabla_{\rho}^{3}\left(F\left(e^{i t \xi+\rho}\right)\right)(i \xi)^{3} / 3 !
$$


and where $H_{z}=\nabla^{2} F\left(|z|^{2}\right)=\nabla^{2} \varphi\left(e^{\rho}\right)$ is the Hessian in the notation (3.6). Hence, (5.3) takes the form

$$
\begin{aligned}
& \mathcal{N}_{h^{N}}(f)(x) \\
& \quad \sim \int_{\mathbb{R}^{m}} \hat{f}(\xi) e^{i\langle\mu(z), \xi\rangle} e^{(i N)^{-1}\left\langle H_{z} \xi, \xi\right\rangle+N^{-1} R_{3}(\xi, N, z)} A_{N}\left(z, e^{i(\xi / N)} z, 0, N\right) d \theta
\end{aligned}
$$

and by Taylor expanding, the factor $e^{(i N)^{-1}\left\langle H_{z} \xi, \xi\right\rangle+N^{-1} R_{3}(\theta, N, z)}$, one obtains an amplitude $\tilde{A}_{N}$ such that

$$
\mathcal{N}_{h^{N}}(f)(x) \sim \int_{\mathbb{R}^{m}} \hat{f}(\xi) e^{i\langle\mu(z), \xi\rangle} \tilde{A}_{N}\left(z, e^{i(\xi / N)} z, 0, N\right) d \theta .
$$

The amplitude $\tilde{A}_{N}$ has an expansion of the form,

$$
\tilde{A}_{N}\left(z, e^{i(\xi / N)} z, 0, N\right)=N^{m} a_{0}+N^{m-1} a_{1}+O\left(N^{m-1}\right),
$$

for various smooth coefficients $a_{j}(z)$; the first one is constant. If we divide by $\Pi_{h^{N}}(z, z)$ we cancel the constant and by expanding the denominator we obtain,

$$
\begin{aligned}
\mathcal{N}_{h^{N}}(f)(x) \sim & \left(\frac{N}{\pi}\right)^{m}\left(f(\mu(z))+N^{-1}\left(i^{-1}\left\langle H_{z} D_{x}, D_{x}\right\rangle f(\mu(z))\right.\right. \\
& \left.\left.+a_{1}(z, z) f(\mu(z))\right)+O\left(N^{-2}\right)\right) .
\end{aligned}
$$

As a check on the leading term, we set $f \equiv 1$ and use Remark 4.2. Since $\mu(z)=x$, we obtain Theorem 1.2. Dividing by $\Pi_{h^{N}}(z, z)$ and using (5.1) completes the proof of Theorem 1.1.

It is difficult (but possible) to calculate the coefficients in explicit geometric terms by this method. In the next section, we will reduce the calculation to the known calculation of Bergman kernel expansion coefficients.

\section{Calculation of coefficients for the Bernstein expansion of Theorems 1.1 and $\mathbf{1 . 2}$}

In this section, we give a second proof of Theorems 1.1 and 1.2 which gives a more effective approach to the calculation of the coefficients in the Bernstein polynomimal expansion, and in particular we calculate the operator $\mathcal{N}_{1}$ in the expansion of Theorem 1.2. The approach is based on the localization of the sum over $\frac{\alpha}{N} \in P \cap \frac{1}{N} \mathbb{Z}^{m}$ around the image of $z$ under the moment map. For classical Bernstein polynomials, this is well-known and various expositions can be found in $[\mathbf{1 8}, \mathbf{2 0}, \mathbf{2 1}]$; see also [10] Lemma 6.3.5). The localization approach reduces the calculation of the lower order terms in the Bernstein polynomial expansion to that of the Bergman kernel expansion in $[\mathbf{2 2}, \mathbf{3 2}]$ and elsewhere. 
The relevant localization lemma was proved in $[\mathbf{2 6}]$. We use a notation similar to $[\mathbf{1 8}]$.

Lemma 6.1 (Localization of Sums [26]). Let $f \in C(\bar{P})$. Then, there exists $C>0$ so that

$$
\begin{aligned}
\sum_{\alpha \in N P \cap \mathbb{Z}^{m}} f\left(\frac{\alpha}{N}\right) \frac{\left|S_{\alpha}(z)\right|_{h^{N}}^{2}}{\mathcal{Q}_{h^{N}}(\alpha)}= & \sum_{\substack{\alpha\left|(\alpha / N)-\mu_{h}(z)\right| \leq N^{-1+\delta}\\
}} f\left(\frac{\alpha}{N}\right) \frac{\left|S_{\alpha}(z)\right|_{h^{N}}^{2}}{\mathcal{Q}_{h^{N}}(\alpha)} \\
& +O_{\delta}\left(N^{-C}\right) .
\end{aligned}
$$

Given the localization lemma, it is natural to Taylor expand $f$ around $\mu_{h}(z)$ to obtain

$$
f\left(\frac{\alpha}{N}\right)=\sum_{\nu<2 M} f^{(\nu)}\left(\mu_{h}\left(e^{\rho}\right)\right)\left(\frac{\alpha}{N}-\mu_{h}\left(e^{\rho}\right)\right)^{\nu} / \nu !+R_{M}\left(f, e^{\rho}, \frac{\alpha}{N}\right),
$$

where $R_{M}$ is the $M$ th order Taylor remainder. We then have,

$$
\begin{aligned}
\mathcal{N}_{h^{N}} f(x)= & \sum_{\beta:|\beta| \leq M} \frac{1}{\beta !} D_{x}^{\beta} f(\mu(z))\left(\sum_{\alpha \in N \mathbb{P} \cap \mathbf{Z}}\left(\frac{\alpha}{N}-\mu_{h}(z)\right)^{\beta} \frac{\left|S_{\alpha}\right|_{h^{N}}^{2}}{\mathcal{Q}^{N}(\alpha)}\right) \\
& +\mathcal{R}(M, N, z),
\end{aligned}
$$

where the remainder is obtained by summing $R_{M}\left(f, e^{\rho}, \frac{\alpha}{N}\right)$ in the variable $\frac{\alpha}{N}$.

The next step is to study the special functions

$$
\begin{aligned}
I_{h^{N}}^{\nu}(z) & :=\sum_{\alpha \in N \mathbb{P} \cap \mathbf{Z}}\left(\frac{\alpha}{N}-\mu_{h}(z)\right)^{\nu} \frac{\left|S_{\alpha}(z)\right|_{h^{N}}^{2}}{\mathcal{Q}_{h}^{N}(\alpha)} \\
& =\sum_{\alpha \in \mathbb{P} \cap \mathbf{Z}}\left(\frac{\alpha}{N}-\mu_{h}\left(e^{\rho / 2}\right)\right)^{\nu} \frac{e^{\langle\alpha, \rho\rangle-N \varphi_{t}\left(e^{\rho / 2}\right)}}{\mathcal{Q}_{h}^{N}(\alpha)} .
\end{aligned}
$$

Proposition 6.2. Uniformly for $z \in M$, we have

$$
I_{h^{N}}^{\nu}(z)=O\left(N^{m-\nu / 2}(\log N)^{\nu}\right) .
$$

Proof. The localization lemma implies that

$$
I_{h^{N}}^{\nu}(z)=\sum_{\alpha \in N P \cap \mathbb{Z}^{m}:\left|\frac{\alpha}{N}-\mu_{h}(z)\right| \leq \frac{C \log N}{N}}\left(\frac{\alpha}{N}-\mu_{h}(z)\right)^{\nu} \frac{\left|S_{\alpha}(z)\right|_{h^{N}}^{2}}{Q^{N}(\alpha)}+O\left(N^{-C}\right) .
$$


In the domain of summation, we then have

$$
\left(\frac{\alpha}{N}-\mu_{h}\left(e^{\rho / 2}\right)\right)^{\nu}=\left(\frac{\log N}{\sqrt{N}}\right)^{\nu}
$$

and this implies the statement.

We can explicitly evaluate these functions by relating them to derivatives of the Bergman-Szegö kernels. The following lemma was also used in [26]. We employ a tensor product notation $\left(\frac{\alpha}{N}-\mu_{h}\left(e^{\rho / 2}\right)\right)_{i j}^{\otimes 2}$ for $\left(\frac{\alpha_{i}}{N}-\right.$ $\left.\mu_{h}\left(e^{\rho / 2}\right)_{i}\right)\left(\frac{\alpha_{j}}{N}-\mu_{h}\left(e^{\rho / 2}\right)_{j}\right)$. In the following, we implicitly assume that $z$ lies in the open orbit and express it as $z=e^{\rho / 2+i \theta}$. Similar formula holds at the boundary as well where the vector fields $\frac{\partial}{\partial \rho_{j}}$ are replaced by derivatives in affine coordinates. For the sake of brevity, we refer to [26] for the modifications to the formulae around the boundary.

Proposition 6.3. We have:

(1) $\sum_{\alpha \in N P \cap \mathbb{Z}^{m}}\left(\frac{\alpha}{N}-\mu\left(e^{\rho / 2}\right)\right) \frac{e^{\langle\alpha, \rho\rangle-N \varphi\left(e^{\rho / 2}\right)}}{\mathcal{Q}_{h^{N}}(\alpha)}=\frac{1}{N} \nabla_{\rho} \Pi_{h^{N}}\left(e^{\rho / 2}, e^{\rho / 2}\right)$;

(2) $\sum_{\alpha \in N P \cap \mathbb{Z}^{m}}\left(\frac{\alpha}{N}-\mu\left(e^{\rho / 2}\right)\right)_{i j}^{\otimes 2} \frac{e^{\langle\alpha, \rho\rangle-N \varphi\left(e^{\rho / 2}\right)}}{\mathcal{Q}_{h^{N}}(\alpha)}=\frac{1}{N} \Pi_{h^{N}}\left(e^{\rho / 2}, e^{\rho / 2}\right) \nabla_{\rho}^{2} \varphi$ $+\frac{1}{N^{2}} \nabla^{2} \Pi_{h^{N}}\left(e^{\rho / 2}, e^{\rho / 2}\right)$.

Proof. To prove (1.1), we differentiate (3.3) to obtain

$$
\begin{aligned}
\nabla_{\rho} \Pi_{h^{N}}\left(e^{\rho / 2}, e^{\rho / 2}\right)= & N \sum_{\alpha \in N P \cap \mathbb{Z}^{m}}\left(\frac{\alpha}{N}-\mu\left(e^{\rho / 2}\right)\right) \\
& \times \frac{e^{\langle\alpha, \rho\rangle-N \varphi\left(e^{\rho / 2}\right)} \Pi_{h^{N}}\left(e^{\rho / 2}, e^{\rho / 2}\right)}{\mathcal{Q}_{h^{N}}(\alpha)} .
\end{aligned}
$$

To prove (1.2), we take a second derivative of (1.1) in $\rho$ to get

$$
\begin{aligned}
\nabla_{\rho}^{2} \Pi_{h^{N}}\left(e^{\rho / 2}, e^{\rho / 2}\right)= & \left.-N \nabla \mu_{h}\left(e^{\rho / 2}\right)\right) \Pi_{h^{N}}\left(e^{\rho / 2}, e^{\rho / 2}\right) \\
& +N^{2} \sum_{\alpha \in N P \cap \mathbb{Z}^{m}}\left(\frac{\alpha}{N}-\mu_{h}\left(e^{\rho / 2}\right)\right)^{\otimes 2} \frac{e^{\langle\alpha, \rho\rangle-N \varphi\left(e^{\rho / 2}\right)}}{\mathcal{Q}_{h^{N}}(\alpha)} .
\end{aligned}
$$

We now evaluate these functions geometrically. Recall that $S$ is the scalar curvature of the Kähler metric $\omega_{h}$. Below, $C_{m}$ denotes a constant depending only on the dimension (which may vary in each occurrence).

Proposition 6.4. We have

(1) $I_{h^{N}}^{(1)}(z)=C_{m} N^{m-2} \nabla S(z)+O\left(N^{m-3}\right)$;

(2) $I_{h^{N}}^{(2)}(z)=\pi^{-m} N^{m-1} \nabla_{\rho}^{2} \varphi+\frac{1}{2} N^{m-2} S(z) \nabla_{\rho}^{2} \varphi+O\left(N^{m-3}\right)$. 
Proof. From (5.1) it follows that

$$
\begin{aligned}
\nabla_{\rho} \Pi_{h^{N}}(z, z) & =\pi^{-m} N^{m-1} \frac{1}{2} \nabla S(z)+O\left(N^{m-2}\right), \\
\nabla_{\rho} \mu_{h}(z) \Pi_{h^{N}}(z, z) & =\pi^{-m} N^{m}\left(\nabla \mu_{h}+\frac{1}{2} N^{-1} S(z) \nabla \mu_{h}(z)+O\left(N^{-2}\right)\right) ; \\
\nabla_{\rho}^{2} \Pi_{h^{N}}\left(e^{\rho / 2}, e^{\rho / 2}\right) & =\frac{1}{2} \pi^{-m} N^{m-1} \nabla_{\rho}^{2} S(z)+O\left(N^{m-2}\right) .
\end{aligned}
$$

We also use that $\nabla \mu_{h}\left(e^{\rho / 2}\right)=\nabla^{2} \varphi$.

To complete the second proof of Theorem 1.1, it suffices to observe that the remainder in (6.1) after expanding to order $M$ is $O\left(N^{m-M / 2}(\log N)^{M}\right)$, which follows from the fact that $\mathcal{R}(M, N, z) \leq C_{f} N^{m} I_{h^{N}}^{\nu+1}(z)$.

6.1. Calculation of $\mathcal{N}_{1}$. By Proposition 6.4 and by (5.1) and (5.2),

$$
\begin{aligned}
\mathcal{N}_{h^{N}}(f)(\mu(z))= & f(\mu(z)) \Pi_{h^{N}}(z, z) \\
& +\sum_{|\beta|=1} D^{\beta} f(\mu(z)) I_{h^{N}}^{(\beta)}(\mu(z)) \\
& +\frac{1}{2} \sum_{|\beta|=2} D^{\beta} f(\mu(z)) I_{h^{N}}^{\beta}(\mu(z))+O\left(N^{-3 / 2}(\log N)^{3}\right) \\
= & \left(\frac{N}{\pi}\right)^{m}\left(f(\mu(z))+\frac{N^{-1}}{2}\left(f(\mu(z)) S(z)+\nabla \mu_{h}(z) \cdot \nabla^{2} f(\mu(z))\right)\right. \\
& +O\left(N^{m-3 / 2}(\log N)^{3}\right) .
\end{aligned}
$$

After multiplying by $\pi^{m}$ we obtain the stated result.

\section{Dedekind-Riemann sums over lattice points: Proof of Corollary 1.3}

To prove the corollary, we integrate the expansion (5.8) over $P$. The terms in

$$
\left.\left(\hat{\Pi}_{h^{N}} f\left(N^{-1} D_{\theta}\right) \hat{\Pi}_{h^{N}}\right)\left(e^{i \theta} z, z\right)\right|_{\theta=0}
$$

are the values on the diagonal of an orthonormal basis of $H^{0}\left(M, L^{N}\right)$. When integrated over $M$ one obtains $\sum_{\alpha \in N P} f\left(\frac{\alpha}{N}\right)$. Since (7.1) is constant along torus orbits, and since $\frac{\omega_{h}^{m}}{m !}$ is a constant multiple of $d \theta d x$, we obtain

$$
\sum_{\alpha \in N P} f\left(\frac{\alpha}{N}\right)=\pi^{m} \int_{P} \mathcal{N}_{h^{N}}(f)(x) d x .
$$

Here, we use that $\pi^{m}$ is the common volume of the torus fibers. We can calculate this constant by putting $f \equiv 1$ and determining the leading order 
term in the asymptotics as $N \rightarrow \infty$. The left side is $\#\left\{\alpha \in N P \cap \mathbb{Z}^{m}\right\} \sim$ $N^{m} \operatorname{Vol}(P)+\cdots$ while the right side is $C_{L}\left(\frac{N}{\pi}\right)^{m} \operatorname{Vol}(P)+\cdots$. Matching expressions shows that the fiber volume is $\pi^{m}$.

The existence of an asymptotic expansion for the Riemann sums thus follows immediately from Theorem 1.2. However, it is an expansion in terms of integrals of curvature invariants against derivatives of $f$ over $P$. We now prove that the first two terms can be put in the form stated in Corollary 1.3, and thus to clarify the relation between the Bernstein and Euler-MacLaurin approaches to lattice point sums.

By Proposition 6.4, only the zeroth and second order terms of the Taylor expansion of $f$ contribute to the $N^{-1}$ term of the Riemann sum expansion, and we have

$$
\begin{aligned}
\sum_{\alpha \in N P} f\left(\frac{\alpha}{N}\right)= & \pi^{m} \int_{P} f(x) \Pi_{h^{N}}\left(\mu^{-1}(x), \mu^{-1}(x)\right) d x \\
& +\frac{\pi^{m}}{2} \sum_{|\beta|=2} \int_{P} D^{\beta} f(x) I_{h^{N}}^{\beta}(x) d x+O\left(N^{-3 / 2}(\log N)^{3}\right) \\
= & N^{m} \int_{P} f(x) d x+N^{m-1} \int_{P}\left(\frac{1}{2} f(x) S\left(\mu^{-1}(x)\right)\right. \\
& +\frac{1}{2}\left\langle\nabla_{\rho} \mu_{h}\left(\mu^{-1}(x), \nabla_{x}^{2} f(x)\right\rangle\right) d x+O\left(N^{m-3 / 2}(\log N)^{3}\right) .
\end{aligned}
$$

Here, $\left\langle\nabla \mu_{h}, \nabla^{2} f(\mu(z))\right\rangle$ denotes the Hilbert-Schmidt inner product of the tensors.

By Legendre duality, the Hessians of the Kähler potential and symplectic potentials are inverses, i.e.,

$$
\nabla_{\rho} \mu_{h}\left(\mu^{-1}(x)=\left(\nabla^{2} u_{\varphi}(x)\right)^{-1} .\right.
$$

Hence,

$$
\left\langle\nabla_{\rho} \mu_{h}\left(\mu^{-1}(x), \nabla_{x}^{2} f(x)\right\rangle d x=\int_{P} \sum_{j k} u_{\varphi}^{j k} f_{, j k} d x .\right.
$$

Further, we recall (cf. $[\mathbf{2}, \mathbf{1 2}]$ ) that the scalar curvature of a toric Kähler metric is given in terms of the symplectic potential by

$$
S=-\sum_{j, k} \frac{\partial^{2} u_{\varphi}^{j k}}{\partial x_{j} \partial x_{k}}
$$

where $u_{\varphi}^{j k}, 1 \leq j, k \leq n$ are the entries of the inverse of the matrix $\nabla^{2} u_{\varphi}$; see $[12,3.1 .4]$.

We now use the following integration by parts formula due to Donaldson: 
Lemma 7.1 ( [12], Lemma 3.3.5). For any symplectic potential $u_{\varphi}$ and $f \in C^{\infty}, \sum_{j k} u_{\varphi}^{j k} f_{, j k} \in L^{1}(P)$ and

$$
\int_{P} \sum_{j k} u_{\varphi}^{j k} f_{, j k}=\int_{P} \sum_{j k}\left(u_{\varphi}^{j k}\right)_{, j k} f d x+\int_{\partial P} f d \sigma,
$$

where $d \sigma$ is the measure defined in Corollary 1.3.

Combining Lemma 7.1 and (7.6) we obtain

$$
\int_{P} \frac{1}{2} f(x) S\left(\mu^{-1}(x)\right)+\frac{1}{2}\left\langle\nabla_{\rho} \mu_{h}\left(\mu^{-1}(x), \nabla_{x}^{2} f(x)\right\rangle d x=\frac{1}{2} \int_{\partial P} f d \sigma,\right.
$$

proving that the two term expansion in Corollary 1.3 is correct.

Remark 7.2. (i) We note that in [12, Lemma 3.3.5], the boundary term is given the - sign. However, the measure $d \sigma$ was only defined there (page 307) up to sign. The sign of this term is universal and by comparing with the one-dimensional case, we see that it is positive.

(ii) To connect this calculation to the classical one-dimensional case (2.3), and perhaps clarify the notation, we note that its $N^{m-1}$ term (with $m=1$ ),

$$
\int_{0}^{1} f(x) d x+\frac{1}{2} \int_{0}^{1}\left(x-x^{2}\right) f^{\prime \prime}(x) d x
$$

may be expressed in terms of the Fubini-Study Kähler potential and moment map as

$$
\int_{0}^{1} \frac{d}{d \rho} \mu_{\mathrm{FS}}\left(\mu^{-1}(x)\right) f^{\prime \prime}(x) d x, \quad x=\mu\left(e^{\rho / 2}\right)
$$

since

$$
\varphi_{\mathrm{FS}}\left(e^{\rho / 2}\right)=\log \left(1+e^{\rho}\right), \frac{d}{d \rho} \varphi_{\mathrm{FS}}\left(e^{\rho / 2}\right)=\mu_{\mathrm{FS}}\left(e^{\rho / 2}\right)=\frac{e^{\rho}}{1+e^{\rho}}=x,
$$

and

$$
\frac{d^{2}}{d \rho^{2}} \varphi_{\mathrm{FS}}\left(e^{\rho / 2}\right)=\frac{e^{\rho}}{\left(1+e^{\rho}\right)^{2}}=x(1-x) .
$$

Regarding $S$, we recall that it is the scalar curvature of the metric $g_{1 \overline{1}}$ associated to the Kähler form $\omega_{\mathrm{FS}}=\frac{i}{2} \partial \bar{\partial}\left(1+|z|^{2}\right)$, thus

$$
S=-\frac{\partial^{2}}{\partial z \partial \bar{z}} \log \left(1+|z|^{2}\right)^{-2}=2 \operatorname{Tr} g_{1 \overline{1}}=2 .
$$

\section{References}

[1] U. Abel and M. Ivan, Asymptotic expansion of the multivariate Bernstein polynomials on a simplex, Approx. Theory Appl. (N.S.) 16(3), (2000), 85-93.

[2] M. Abreu, Khler geometry of toric manifolds in symplectic coordinates. Symplectic and contact topology: interactions and perspectives (Toronto, ON/Montreal, 
QC, 2001), Fields Inst. Commun, 35, Amer. Math. Soc., Providence, RI, 2003, $1-24$.

[3] R. Berman and J. Sjoestrand, Asymptotics for Bergman-Hodge kernels for high powers of complex line bundles, arXiv:math/0511158.

[4] R. Berman, B. Berndtsson and J. Sjoestrand, A direct approach to Bergman kernel asymptotics for positive line bundles, Ark. Mat. 46(2), (2008), 197-217, arXiv:math/0506367.

[5] S. Bernstein, Démonstration du théorème de Weierstrass, fondée sur le calcul des probabilités, Comm. Soc. Math. Kharkov = Charkow Ges. (2) 13(1-2), (1912), JFM 43.0301.03.

[6] - Lecons sur les proprits extrḿales et la meilleure approximation des fonctions analytiques d'une variable rèele, Gauthier-Villars, 1926.

[7] Collected Works: Volume I: Constructive Theory of Functions, Translation Series U. S. Atomic Energy Commission AEC-tr-3460 (1952).

[8] L. Boutet de Monvel and J. Sjöstrand, Sur la singularité des noyaux de Bergman et de Szegö, Asterisque 34-35 (1976), 123-164.

[9] L. Charles, Berezin-Toeplitz operators, a semi-classical approach, Comm. Math. Phys. 239(1-2) (2003), 1-28.

[10] P. J. Davis, Interpolation and approximation, Dover Publications, Inc., New York, 1975.

[11] S.K. Donaldson, Scalar curvature and projective embeddings, I, J. Diff. Geom. 59 (2001), 479-522.

[12] - Scalar curvature and stability of toric varieties, J. Differential Geom. 62(2) (2002), 289-349.

[13] - Kähler geometry on toric manifolds, and some other manifolds with large symmetry, arXiv:0803.0985.

[14] R. Feng, Szasz analytic functions and toric varieties, preprint, 2008.

[15] V. Guillemin, Kaehler structures on toric varieties, J. Diff. Geom. 40(2) (1994), 285-309.

[16] V. Guillemin and S. Sternberg, Riemann sums over polytopes, math.CO/0608171.

[17] V. Guillemin, S. Sternberg and J. Weitsman, The Ehrhart Function for Symbols, arXiv:math/0601714.

[18] L. Hörmander, The multinomial distribution and some Bergman kernels, in 'Geometric Analysis of PDE and Several Complex Variables', Contemp. Math., 368, 249-265 Amer. Math. Soc., Providence, RI, 2005.

[19] Y. Karshon, S. Sternberg and J. Weitsman, Euler-Maclaurin with remainder for a simple integral polytope, Duke Math. J. 130(3) (2005), 401-434.

[20] E. Kowalski, Bernstein polynomials and Brownian motion, Amer. Math. Monthly 113(10) (2006), 865-886.

[21] G.G. Lorentz, Bernstein polynomials, 2nd ed., Chelsea Publishing Co., New York, 1986.

[22] Z. Lu, On the lower order terms of the asymptotic expansion of Tian-Yau-Zelditch, Amer. J. Math. 122(2) (2000), 235-273.

[23] D.H. Phong and J. Sturm, The Monge-Ampère operator and geodesics in the space of Kähler potentials, Invent. Math. 166(1) (2006), 125-149, arxiv:math.DG/0504157, 2006. 
[24] B. Shiffman, T. Tate and S. Zelditch, Harmonic analysis on toric varieties, in 'Explorations in Complex and Riemannian Geometry', Contemp. Math., 332, 267-286, Amer. Math. Soc., Providence, RI, 2003.

[25] J. Song and S. Zelditch, Convergence of Bergman geodesics on $\mathbf{C P}^{1}$, Annales Institut Fourier, 57(7) (2007), 2209-2237.

[26] - Bergman metrics and geodesics in the space of Kähler metrics on toric varieties, arXiv:0707.3082v2.

[27] - Test configurations and geodesic rays in hermitian metrics on toric varieties, arXiv:0712.3599.

[28] G. Szekelyhidi, Extremal metrics and K-stability, Thesis Imperial College of London, arXiv:math.DG/0611002.

[29] T. Tate, Bernstein measures on convex polytopes, to appear in 'Spectral Analysis in Geometry and Number Theory on the occasion of Toshikazu Sunada's 60th birthday', Contemp. Math. Series.

[30] T. Tate and S. Zelditch, Lattice path combinatorics and asymptotics of multiplicities of weights in tensor powers, J. Funct. Anal. 217(2) (2004), 402-447.

[31] G. Tian, On a set of polarised Kähler metrics on algebraic manifolds, Jour. Differential Geometry 32 (1990) 99-130.

[32] S. Zelditch, Szegö kernels and a theorem of Tian, IMRN 6 (1998), 317-331.

Department of Mathematics

JOHNS HOPKINS UNIVERSITY

BALTIMORE

MD 21218

USA

E-mail address: zelditch@math.jhu.edu

Received 8/2/07, accepted 9/18/08

Research partially supported by NSF grant DMS-0603850. 